\title{
MicroRNA-223 demonstrated experimentally in exosome-like vesicles is associated with decreased risk of persistent pain after lumbar disc herniation
}

\author{
Aurora Moen ${ }^{1,2 \dagger}$, Daniel Jacobsen ${ }^{1 \dagger}$, Santosh Phuyall ${ }^{1}$, Anna Legfeldt ${ }^{1}$, Fred Haugen ${ }^{1}$, Cecilie Røe 2,3
} and Johannes Gjerstad ${ }^{1,4^{*}}$

\begin{abstract}
Background: Previous findings have demonstrated that lumbar radicular pain after disc herniation may be associated with up-regulation of inflammatory mediators. In the present study we examined the possible role of extracellular microRNAs (miRs) in this process.

Methods: Single unit recordings, isolation of exosome-like vesicles, electron microscopy, nanoparticle tracking analysis, western blot analysis and qPCR were used in rats to demonstrate the effect of nucleus pulposus (NP) applied onto the dorsal nerve roots. ELISA and GPCR were used to measure the level of circulating IL-6 and miRs in a 1-year observational study in patients after disc herniation.

Results: In the rats, enhanced spinal cord nociceptive responses were displayed after NP applied onto the dorsal nerve roots. An increased release of small non-coding RNAs, including miR-223, miR-760 and miR-145, from NP in exosome-like vesicles was demonstrated. In particular, the NP expression of miR-223, which inhibited the nociceptive spinal signalling, was increased. In the patients, increased extracellular miR-223 was also verified in the acute phase after disc herniation. The increased miR-223 expression was, however, only observed in those who recovered (sex, age and smoking were included as covariates).
\end{abstract}

Conclusions: Our findings suggest that miR-223, which can be released from the NP after disc herniation, attenuates the neuronal activity in the pain pathways. Dysregulation of miR-223 may predict chronic lumbar radicular pain.

Trial registration/ethics REK 2014/1725

Keywords: Lumbar radicular pain, Disc herniation, Inflammation, Immune response, microRNA-223, miR-223

\section{Background}

Experimental data suggest that lumbar disc herniation may induce sensitization of the primary afferent nerve fibers, even in the absence of nerve root compression [1-5]. The local release of inflammatory molecules, such as interleukin-6 (IL-6), nitric oxide (NO), prostaglandin

\footnotetext{
*Correspondence: Johannes.Gjerstad@stami.no

${ }^{\dagger}$ Aurora Moen and Daniel Jacobsen contributed equally to this work

${ }^{1}$ National Institute of Occupational Health, Pb 8149 Dep., 0033 Oslo, Norway

Full list of author information is available at the end of the article
}

E2 (PGE2) and matrix metalloproteinases (MMPs) [6], may be a crucial part of this process. For instance, the interaction between the nucleus pulposus (NP) cells and recruited immune cells may up-regulate IL-6 and MMPs $[7,8]$. The circulating cytokine levels are also elevated in patients with moderate or severe pain following disc herniation $[9,10]$.

Such local or systemic inflammatory processes can be modulated by specific intracellular microRNAs (miRs), i.e., small non-coding RNAs that act by binding to the 3'UTR (untranslated region) of target mRNA, resulting in either translational repression or mRNA degradation 
[11]. Previous data suggest that miR-145 may regulate immune cells [12], and that inflammatory responses in the intervertebral disc may be reduced by miR-146a [13]. Moreover, decreased miR-155 and increased miR-27a in the disc may be associated with apoptosis [14, 15], whereas up-regulation of miR-10b and miR-21 is linked to NP cell proliferation $[16,17]$.

Interestingly, biologically active miR-223 [18] can be exchanged between cells via exosomes, which are small extracellular micro-vesicles that contain RNA and protein cargos [19]. Therefore, miRs released in exosomes may affect intracellular signalling in adjacent cells [20, 21]. Some extracellular miRs can also directly interact with cell-surface receptors [22]. For example, miR-let-7b may directly activate nociceptive nerve fibers and elicit pain via TLR7 and TRPA1 [23]. Additionally, extracellular miRs are present in human serum and plasma, where they appear to be resistant to RNAase degradation. A number of protein-encoding genes may be regulated by miRs [24].

In patients, down-regulated circulating miR expression has been reported in migraines [25], complex regional pain syndrome [26], fibromyalgia [27] and rheumatoid arthritis [28]. Hence, we hypothesized that miRs could be involved in inflammatory processes and influence the risk of persistent pain after disc herniation. The present study suggests that miR-223 may be linked to the recovery rate in lumbar radicular pain patients.

\section{Methods}

\section{Animals and surgery}

Inbred female Lewis rats were used in the experiments. The animals were sedated with isoflurane gas and anesthetized with urethane ( 2 $\mathrm{g} / \mathrm{kg}$ bodyweight, i.p.). Absence of paw withdrawal and ear reflex to pinch indicated adequate surgical anaesthesia. The rat core temperature was maintained at $36-37{ }^{\circ} \mathrm{C}$ with a feedback heating pad. A laminectomy, with a 1-mm lateral expansion on the left side for applying NP, was performed at vertebrae Th13-L1, corresponding to spinal cord segments L3-S1. The vertebral column was rigidly fixed by clamps rostral and caudal to the exposed spinal cord segments. The meninges, i.e., dura mater and arachnoidea, were punctured by a cannula and carefully removed by two tweezers. A section of $8-10 \mathrm{~mm}$ of the sciatic nerve was freed at the mid-thigh level and isolated from the surrounding tissue by a plastic film. A caudectomy was performed on genetically identically donor rats, and NP was harvested from 3 to 8 caudal intervertebral discs.

All animal experiments were approved by the Norwegian Animal Research Authority (NARA) and were performed in conformity with the laws and regulations controlling experiments and procedures on live animals in Norway. The rats were euthanized immediately after the end of the experiments.

\section{Electrophysiological recordings}

A bipolar silver hook electrode $(1.5 \mathrm{~mm}$ between the hooks) was placed proximal to the main branches of the sciatic nerve for electrical stimulation. A parylene-coated tungsten microelectrode with impedance of 2-4 $\mathrm{M} \Omega$ (Frederick Haer \& Co, Bowdoinham, USA) was lowered into the left dorsal horn by an electrically controlled micromanipulator (Märzhäuser Wetzlar $\mathrm{GmbH} \&$ Co. KG, Wetzlar, Germany), and a reference electrode was placed subcutaneously. Extracellular single cell recordings were performed at depths of $100-600 \mu \mathrm{m}$ from the surface of the spinal cord [29]. Only one cell was studied in each animal. The recorded signals were amplified, bandpass filtered with a half amplitude cut of 500-1250 Hz (Digitimer Ltd, Hertfordshire, UK), digitalized using a CED $1401 \mu$ interface and displayed on a computer screen by means of the CED Spike 2.2 software (Cambridge Electronic design, Cambridge, UK). The sampling frequency was $20 \mathrm{kHz}$.

The A- and C-fiber responses were separated according to latencies, where spikes $50-300 \mathrm{~ms}$ after stimulus were defined as $\mathrm{C}$-fiber responses. Single cell recordings were ensured by the amplitude and shape of the action potentials. Every 4 th $\mathrm{min}$ a single test stimulus $(2 \mathrm{~ms}$ rectangular pulse, $1.5 \times \mathrm{C}$-fiber threshold) was applied to the sciatic nerve through the hook electrode. A pulse buffer connected to a stimulus isolator unit (NeuroLog System, Digitimer Ltd, Hertfordshire, UK) controlled the stimulus intensity. The C-fiber threshold was defined at the beginning of each experiment as the lowest stimulus intensity necessary to evoke a C-fiber response. Six stable C-fiber responses, varying less than $20 \%$, served as a baseline for the subsequent experiments. Cells were rejected if the C-fiber response consisted of fewer than five or more than 20 spikes at the start of the recording.

Nucleus pulposus was harvested from 3 to 8 caudal vertebrae of genetically identical donor rats and carefully applied caudally to the recording electrode, covering the incoming spinal dorsal nerve roots [30]. The C-fiber responses were followed for $180 \mathrm{~min}$ after application of $\mathrm{NP}$ transplant covering $1-2 \mathrm{~mm}$ of the dorsal nerve roots, or $40 \mu \mathrm{L} 0.6 \mathrm{mg} / \mathrm{mL}$ miR-223-3p in Invivofectamine (Invitrogen, Carlfbad, USA). In accordance with our earlier studies [30,31], NP was applied in absence of nerve root compression. Application of $40 \mu \mathrm{L} 0,9 \% \mathrm{NaCl}$ served as controls. 


\section{mRNA analysis}

As previously described [30], total RNA (RIN values were $>7$ ) was isolated from frozen NP tissue and converted into cDNA. The qPCR was performed in two parallels on a StepOnePlus qPCR machine (Applied Biosciences, USA). IL-6 primers (FW; TGCCCTTCAGG AACA; RV; AAGGCAGTGGCTGTC) were designed using Primer Express 2.0 (Applied Biosystems, California, USA) and checked for specificity by performing a BLAST search. Effort was made to design primers without nonspecific binding (the melting curves indicated no biproducts). Target genes were normalized to $\beta$-actin (FW; CTAAG GCCAA CCGTG AAAAG A, RV; ACAAC ACAGC CTGGA TGGCT A) (internal reference).

\section{miR analysis}

After transcardial perfusion with HBSS, NP tissue was harvested from 3 to 8 caudal vertebrae from the same donor rat and then bisected. One piece was immediately frozen after caudectomy ( $\left.\mathrm{NP}^{\text {nativ }}\right)$, and the other piece was applied onto the spinal dorsal nerve roots for 180 min before it was frozen (NPexposed $)$. For the NP graft in media, the tail of a donor rat was cut off at the base, skinned and placed in a sterile petri dish. The NP tissue was harvested from the caudal vertebrae in a LAF bench. The tissue was incubated in $0.5 \mathrm{~mL}$ of medium in a humidified $5 \% \mathrm{CO}_{2}$ incubator at $37{ }^{\circ} \mathrm{C}$. Two groups of samples were provided, differing in the time spent in the incubator $-5 \mathrm{~min}$ and $3 \mathrm{~h}$. After incubation, the samples were spun first at $300 \mathrm{~g}$ and then at $1000 \mathrm{~g}$ in order to retain medium without any cells. miR extraction was performed on $200 \mu \mathrm{L}$ of medium. In accordance with the manufacturer's protocol, total RNA was isolated from frozen $\left(-80{ }^{\circ} \mathrm{C}\right)$ tissue or $200 \mu \mathrm{L}$ of medium using the miRNeasy micro kit (Qiagen) and converted into cDNA using the miScript $\mathrm{HiSpec}$ reverse transcription buffer (Qiagen).

\section{qPCR array}

The screening of miRs was performed using the Rat Pain: Neuropathic and Inflammatory MiScript miR PCR Array containing 84 miRs (cat.no. MIRN-120Z, Qiagen). cDNA from $\mathrm{NP}^{\text {nativ }}$ and $\mathrm{NP}^{\text {exposed }}$ were separately pooled before the analyses. Normalization was performed to the mean of all six snoRNA/snRNAs controls included in each array.

Based on the array analysis, miRs with a fold change $>5$ were selected for follow-up analysis with qPCR. In this case, the mean of the SNORD61 and SNORD68 reference genes was used for normalization of gene expression. All primers were predesigned and delivered by Qiagen. Then,
$1 \mathrm{ng}$ of cDNA was used in the qPCR reaction. qPCR was performed on an Applied Biosystems 7900 Real Time PCR System with the following conditions: $95{ }^{\circ} \mathrm{C}$ for $15 \mathrm{~min}$, followed by 40 cycles at $95{ }^{\circ} \mathrm{C}$ for $5 \mathrm{~s}, 55^{\circ} \mathrm{C}$ for $30 \mathrm{~s}$, and $70{ }^{\circ} \mathrm{C}$ for $30 \mathrm{~s}$.

\section{Exosome-like vesicle (ELV) isolation}

Nucleus pulposus grafts were incubated in the serumfree culture media (Ham's F-12 nutrient mixture) at $37{ }^{\circ} \mathrm{C}$ in a humidified $5 \% \mathrm{CO}_{2}$ incubator for $3 \mathrm{~h}$. After incubation, media were centrifuged at $300 \mathrm{~g}$ for $10 \mathrm{~min}$ and then at $1000 \mathrm{~g}$ for $10 \mathrm{~min}$ to remove cells and other larger contaminants. Media were then centrifuged for $45-\mathrm{min}$ at $10,000 \mathrm{~g}$. Pellets were discarded and supernatants were passed through a $0.45-\mu \mathrm{m}$ filter. Supernatants were ultracentrifuged (Optima MAX-XP Benchtop Ultracentrifuge, Beckman Coulter, Brea, CA, USA) at $100,000 \mathrm{~g}$ for $90 \mathrm{~min}$ to pellet ELVs. The pellet was washed once with PBS and centrifuged again at $100,000 \mathrm{~g}$ for another $90 \mathrm{~min}$. The ELV pellets were resuspended in PBS and stored at $-21^{\circ} \mathrm{C}$ for further analyses. As previously described [32], all centrifugation steps were performed at $4{ }^{\circ} \mathrm{C}$.

\section{Electron microscopy}

The ELVs pellets resuspended in PBS were fixed in $4 \%$ formaldehyde $/ 0.2 \%$ glutaraldehyde and placed on formvar-carbon-coated electron microscopy grids. The grids were then washed, and stained with a mixture of methylcellulose and uranylacetate. Samples were observed in a JEOL-JEM 1230 (JEOL Ltd., Tokyo, Japan) at $80 \mathrm{kV}$ and pictures were acquired using a Morada camera and iTEM software (Olympus, Münster, Germany).

\section{Nanoparticle tracking analysis (NTA)}

Nanoparticle tracking analysis was used to determine the size distribution and concentration of the ELV population. Exosome pellets were resuspended in PBS, vortexed thoroughly for $1 \mathrm{~min}$ and loaded into the NS300 instrument (Malvern Instruments, Worcestershire, UK) using the syringe pump. For all measurements, five videos, $60 \mathrm{~s}$ each, were acquired (infusion rate: 20 with camera settings: shutter, 600; gain, 350-450). Recorded data were processed with the NTA 3.0 software to obtain the size distribution and approximate concentration by tracking the centre of each particle under Brownian motion on a frame-by-frame basis.

\section{SDS-PAGE and immunoblotting}

Exosome-like vesicles were lysed in lysis buffer containing $50 \mathrm{mM}$ Tris- $\mathrm{HCl}, 300 \mathrm{mM} \mathrm{NaCl}, 1 \mathrm{mM}$ EDTA, 0.5\% Triton $\mathrm{X}-100(\mathrm{pH} 7.4)$ in the presence of Halt ${ }^{\mathrm{TM}}$ protease 
inhibitor cocktail (ThermoFisher Scientific, Waltham, MA, USA). ELV proteins were separated by SDS-PAGE in 4-20\% TGX $^{\mathrm{TM}}$ precast gels (BioRad, Hercules, CA, USA) after solubilization in laemmli sample buffer. Proteins were transferred to PVDF membranes using a Tranfer-Blot Turbo Transfer Pack (BioRad). Membranes were blocked in 5\% (w/v) non-fat dry milk dissolved in PBS containing $0.1 \%(\mathrm{v} / \mathrm{v})$ Tween-20. Blots were incubated with the specified primary and secondary antibodies. Blots were visualized with Amersham ${ }^{\mathrm{TM}} \mathrm{ECL}^{\mathrm{TM}}$ Prime Western blot detection (GE Healthcare, Little Chalfont, UK) on an Amersham AI600RGB imaging instrument (GE Healthcare).

\section{Antibodies}

The antibodies used for western blot analysis were as follows: mouse anti-tsg101 (Santa Cruz Biotechnology Inc., Dallas, TX, USA); rabbit anti-CD9 (Abcam, Cambridge, UK); rabbit anti-alix (Merck Millipore, Darmstadt, Germany); and HRP-conjugated secondary antibodies (Cell Signalling Technology, Danvers, MA, USA). All antibody dilutions (1:500 for the primary and 1:5000 for the secondary antibodies, respectively) were prepared in blocking solution.

\section{Patients}

Participants with lumbar radicular pain were recruited from Oslo University Hospital (Ullevål) during 20072009. A total of 122 patients were included in the intended follow-up assessment, $8 \%$ were lost during the follow-up, and 112 patients were ultimately assessed at the 12-month follow-up. All participants received written information and signed an informed consent form. The study was approved by the Norwegian Social Science Data Services and Norwegian Regional Committee for Medical Research Ethics (REK 214/1725).

The inclusion criteria were patients aged 18-60 years, LDH on magnetic resonance imaging with corresponding radicular pain, and positive straight leg raise test results. A positive straight leg raise test was defined by pain radiating into one or both legs when the examiner slowly raised the straightened limb until $60^{\circ}$. The test was performed in the supine position and supplemented with slight dorsiflexion of the foot. The exclusion criteria were lumbar spinal stenosis, previous spinal surgery for a herniated disc at the same level (to rule out radiating pain from epidural fibrosis), fusion surgery at any level in the lumbar spine, generalized musculoskeletal pain (widespread musculoskeletal pain in three out of four body quadrants), inflammatory rheumatic disease, diabetic polyneuropathy, cardiovascular disease (New York Heart Association III and IV), cancer, psychiatric disease, drug abuse and alcoholism, recent surgery (within 1 month), pregnancy, poor proficiency in the Norwegian language, and non-European-Caucasian ethnicity. Patients with cauda equina syndrome were excluded.

\section{Clinical procedure}

At the initial screening visit and 1-year follow-up, the patients underwent a standardized clinical examination, which included an assessment of sensory and motor function, and standardized registrations of pain and function. Pain intensity was recorded using the visual analogue scale (VAS) with anchor values from 0 (no pain) to 10 (worst imaginable pain) over the past week during activity.

Demographic data and the patient's smoking status were obtained upon inclusion in the study. Treatment (surgery or conservative) was determined during the initial screening visit, where surgical treatment was given to patients with persistent radicular pain lasting for more than 8 weeks, neurological deficits (sensory changes, muscle weakness or depressed or absent deep tendon reflexes) and corresponding magnetic resonance imaging findings in the anticipated location. Non-surgical treatment was given to patients who did not clearly meet these criteria. Non-surgical treatment included a brief cognitive intervention, activity guidance during the acute phase of lumbar radicular pain, and physiotherapy for most patients.

\section{Blood sampling and RNA isolation}

At inclusion and 12-month follow-up, venous blood was collected and kept on ice for $45 \mathrm{~min}$. After centrifugation at $2000 \mathrm{~g}$ and $4{ }^{\circ} \mathrm{C}$ and for $10 \mathrm{~min}$, the supernatant serum was collected and stored in aliquots at $-80{ }^{\circ} \mathrm{C}$ until further analysis. Serum was used to analyse the miRs. Samples with visible haemolysis were excluded. Total small RNAs were extracted from $200 \mu \mathrm{L}$ serum using the miRNeasy serum plasma isolation kit (Qiagen) according to the manufacturer's protocol. Synthetic C-elegans (C) miR-39-3p (Qiagen) was spiked-in at a final concentration of $1.6 \times 10^{8}$ copies $/ \mu \mathrm{L}$ after the initial denaturation, prior to extraction, to correct the extraction efficiency. Total RNA was eluted in $14 \mu \mathrm{L}$ of RNase-free water. A fixed volume of $7 \mu \mathrm{L}$ of eluate was used as input for the cDNA synthesis. RNA was converted to cDNA using the qScript microRNA cDNA synthesis kit (Quanta). qPCR was performed with PerfeCta SYBR green supermix on an Applied Biosystems 7900 Real Time PCR System with the following conditions: $95^{\circ} \mathrm{C}$ for $2 \mathrm{~min}$, followed by 40 cycles at $95{ }^{\circ} \mathrm{C}$ for $5 \mathrm{~s}, 60^{\circ} \mathrm{C}$ for $15 \mathrm{~s}$, and $70{ }^{\circ} \mathrm{C}$ for $15 \mathrm{~s}$. Data were normalized to the spiked-in C miR-39-3p.

Of the 112 patients with both baseline and 12-months data, 7 and 3 samples were excluded due to visual 
haemolysis at inclusion and 12 months, respectively. In addition, 1 sample was missing at inclusion, 2 samples were missing at 12 months and 2 samples could not be analysed due to undetectable miR levels at 12 months. In total, this resulted in miR data for both inclusion and 12 months from 97 patients.

\section{Measurements of the cytokine concentration in serum by ELISA-methodology}

As previously described [9], IL-6 concentrations in the serum of the same 97 patients were determined by using commercial ELISA kits (Human ultra-sensitive kits for IL-6, Invitrogen Corporation, CA, USA). Following the manufacturer's instructions, $100 \mu \mathrm{L}$ of the serum samples or standards were added to each well in microtiter plates that were pre-coated with an antibody specific to IL-6 and incubated at room temperature. Serum collected at the two time points for each patient was analysed on the same microtiter plate.

\section{Statistics}

The spinal nociceptive activity, i.e., the C-fiber response, was presented as percent of baseline. Baseline recordings were converted to an average of three consecutive responses, whereas the recordings after NP application were converted to an average of nine consecutive responses, producing two pre-NP/miR-233-3p and five post-NP/ miR-233-3p values. The effect of NP or miR-233-3p on spinal nociceptive response over time was compared to the control by repeated measures analysis of variance (rmANOVA). When the sphericity assumption was not met, a Greenhouse-Geisser correction was applied.

For miR arrays, data analysis was performed using the miScript miR PCR Array Web-based software delivered by Qiagen (http://pcrdataanalysis.sabiosciences.com/ mirna). In brief, $\Delta C_{t}$ value for each miR profiled in the plate is calculated using the formula $\Delta C_{\mathrm{t}}=C_{\mathrm{t}}^{\mathrm{miRNA}}-\mathrm{AVG}$ $C_{\mathrm{t}}^{\mathrm{SN} 1 / 2 / 3 / 4 / 5 / 6}$, where AVG $C_{\mathrm{t}}^{\mathrm{SN} 1 / 2 / 3 / 4 / 5 / 6}$ is the mean of all six snoRNA/snRNAs controls included on each array. $\Delta \Delta C_{\mathrm{t}}$ for each miR across the two groups of samples is calculated using the formula: $\Delta \Delta C_{\mathrm{t}}=\Delta C_{\mathrm{t}}$ (sample) $\Delta C_{\mathrm{t}}$ (control). The fold-change was calculated as $2^{-\Delta \Delta C \mathrm{t}}$.

In the qPCR analysis, fold change values for each sample were defined by the expression of the target gene normalized to the expression of the mean of the reference genes SNORD61 and SNORD68 in the animal studies and C miR-39-3p in the patients. The abundance of each miR was calculated by the comparative $\mathrm{Ct}$ method and $2^{-\Delta \mathrm{Ct}}$ values in animals were analysed by the two-tailed unpaired Student's t test and linear mixed model.
Regarding the clinical arm of the study, the change in IL- 6 and miRs from inclusion to 12 months was analysed by the paired Student's t test. A two-sided Pearson correlation test was performed to examine the relationship between miR-223-3p at inclusion and IL-6 at 12 months and the delta miR values versus delta VAS values. In addition, the miR levels were analyzed by linear regression adjusted for sex, age and smoking. Baseline differences were tested by Pearson Chi square, unpaired Student's t test and Mann-Whitney U test.

\section{Results}

Application of NP onto the dorsal nerve-roots increased the evoked spinal C-fiber responses (Fig. 1a). Most cells reached a plateau within $180 \mathrm{~min}$. However, the C-fiber response in the control group remained at baseline level (Fig. 1b). Over time, a significant increase in the C-fiber response was observed in the NP group (within subject rmANOVA, $\mathrm{p}=0.003$, NP group vs control group). A significant increase in the NP expression of IL- 6 was also observed at $180 \mathrm{~min}$ in the exposed NP tissue compared to native tissue (Fig. 1c).

Analyses of the pooled NP samples using the PCR array demonstrated a more than fivefold increase in the expression of 8 of the 84 miRs in NP following contact with the dorsal nerve roots (Additional file 1: Table S1). Validation with qPCR suggested that 6 of these miRs were more than fivefold up-regulated in exposed NP tissue compared to native NP tissue (Table 1). Interestingly, miR-223-3p, miR-760-5p and miR-145-5p, were also significantly up-regulated after correction for multiple testing (Fig. 2).

To further investigate whether these miRs could be actively released from NP cells, NP was isolated and incubated in vitro (without contact with the nerve). After 5 min and $3 \mathrm{~h}$ of incubation the cells were spun down, and the media were analysed by qPCR. miR-223-3p, miR760-5p and miR-145-5p were also found in the media fraction (Fig. 3). A significant overall increase in the miRs from 5 min to $3 \mathrm{~h}$ was observed [linear mixed model, beta $=0.65, \mathrm{p}=0.001,95 \%$ CI $(0.28,1.01)]$.

Electron microscopy, nanoparticle tracking analysis, western blot analysis and $\mathrm{qPCR}$ were used to demonstrate exosome-like vesicles (ELV) in the media conditioned for $3 \mathrm{~h}$ by NP. The ELVs population in the media appeared as homogenous single vesicles (Fig. 4a). In line with this observation, size distribution analysis with Nanosight (nanoparticle tracking analysis) revealed that most ELVs were between 50 and $100 \mathrm{~nm}$ in diameter, with an estimated mode size of $65 \pm 1.4 \mathrm{~nm}$ (Fig. 4b). 


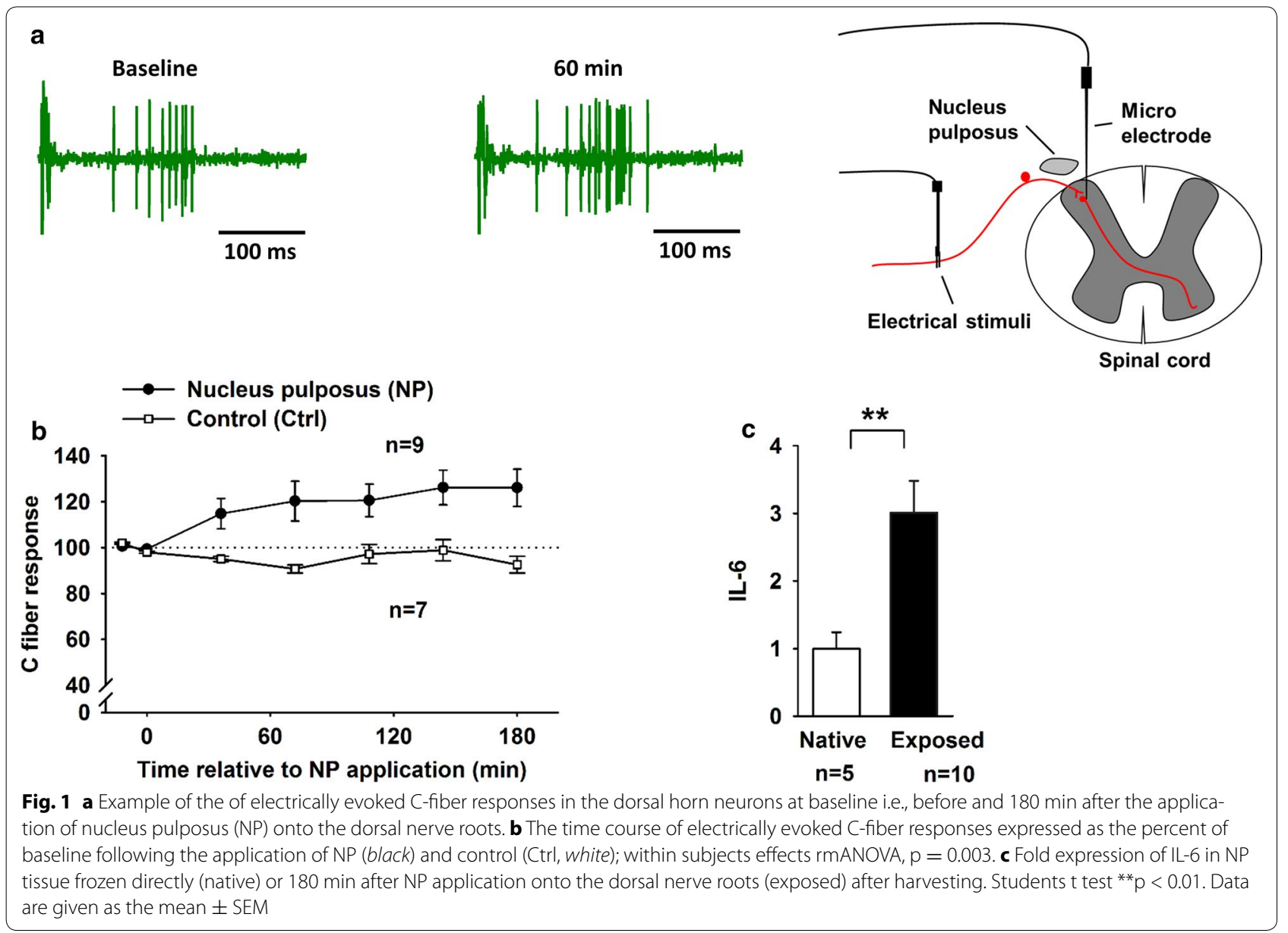

Table 1 miRs more than fivefold up-regulated in exposed NP tissue

\begin{tabular}{lcl}
\hline miRs & Fold change & $\boldsymbol{p}$ value \\
\hline $206-3 p$ & $16.87(5.92)$ & 0.032 \\
$223-3 p$ & $10.10(1.14)$ & $3.1 \times 10^{-5 * *}$ \\
$451-5 p$ & $7.43(1.63)$ & 0.005 \\
$760-5 p$ & $6.34(0.67)$ & $2.3 \times 10^{-5 * *}$ \\
$145-5 p$ & $6.20(0.73)$ & $7.6 \times 10^{-5 * *}$ \\
$142-3 p$ & $5.95(1.28)$ & 0.005 \\
\hline
\end{tabular}

Asterisks represent level of significance after conservative correction (Bonferroni, $\mathrm{n}=84$ ) for multiple testing. ${ }^{* *} \mathrm{p}<0.0001$

The average diameter of the ELVs was estimated to be $88.5 \pm 0.7 \mathrm{~nm}$, thus indicating presence of aggregates and/or larger vesicles.

Moreover, to verify the exosome-like vesicular nature of the obtained pellet, samples were subjected to western blot and blots were probed for specific proteins that are commonly associated with exosomes (Fig. 4c). We detected the presence of alix, tsg-101 and CD9 in our ELV preparations. Additional qPCR analysis of the ELV samples showed that miR-223-3p, miR760-5p and miR145-5p were present in the exosome fraction (Fig. 4d). Application of miR-223-3p onto the spinal nerve roots decreased the $\mathrm{C}$-fiber response in the dorsal horn neurones within $180 \mathrm{~min}$ (Fig. 4e).

Not all patients recovered (Fig. 5a). The serum analyses in patients demonstrated a significant decrease in IL-6 (Fig. 5b) and miR-223-3p (Fig. 5c) from inclusion to 12 months (paired Student's t test, $\mathrm{p}=0.005$ ), which was not the case for miR-760-5p and miR-145-5p (data not shown). No significant association between miR223-3p at inclusion versus IL-6 at 12 months (Fig. 5d) was demonstrated (Pearson's correlation; rho $=-0.04$, $\mathrm{p}>0.05)$.

Further analyses indicated that the change in the miR223-3p expression (Additional file 2: Figure S1) from inclusion to 12 months were correlated with the change 

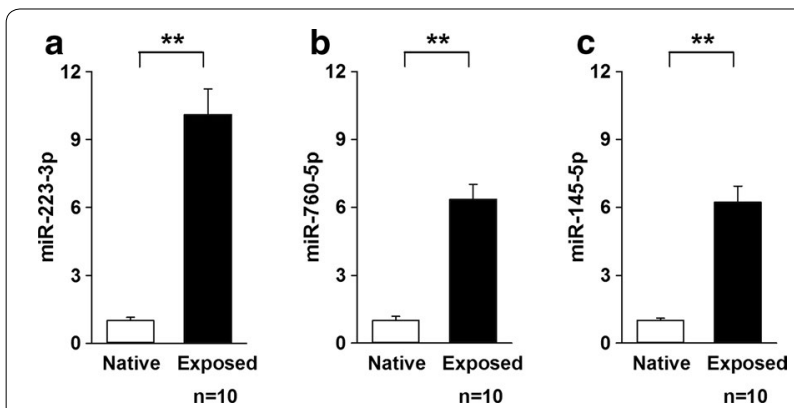

Fig. 2 Fold expression of a miR-223, b miR-760 and $\mathbf{c}$ miR-145 in nucleus pulposus (NP) tissue frozen directly (native) or 180 min after NP application onto the dorsal nerve roots (exposed) after harvesting. Students t test ${ }^{* *} \mathrm{p}<1 \times 10^{-5}$. Data are given as the mean $\pm \mathrm{SEM}$

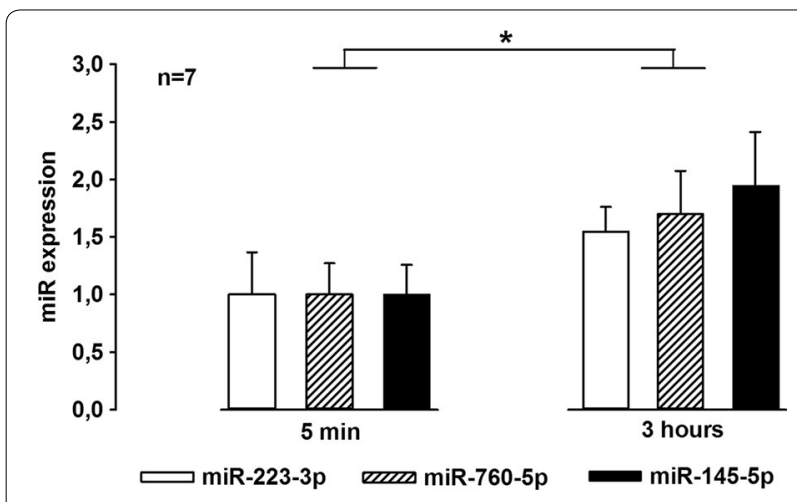

Fig. 3 Fold expression of a miR-223, b miR-760 and $\mathbf{c}$ miR-145 in cellfree medium frozen after nucleus pulposus (NP) incubation for $5 \mathrm{~min}$ or 3 h. Overall linear mixed model, beta $=0.65,{ }^{* *} \mathrm{p}<0.001,95 \% \mathrm{Cl}$ $(0.28,1.01)$

in pain (delta miR values versus delta VAS, two-sided Pearson correlation, $\mathrm{r}=0.221, \mathrm{p}=0.029$ ).

For the final analyses the patients were grouped into recovery and persistent pain groups defined by reduction in VAS score from inclusion to 12 months; recovery group VAS change $>2$ and persistent pain group VAS change $<2$ (Fig. 5e). Differences in the sex, age, treatment and smoking (Table 2) were included as covariates in the analyses. Significantly higher miR-223-3p levels at inclusion were observed in the recovery group than in the persistent pain group [beta $=-2.97, \mathrm{p}=0.031$, $95 \%$ CI $(-5.67,-0.27)]$.

\section{Discussion}

In accordance with earlier observations [30], the application of NP onto the dorsal nerve roots in rats enhanced the spinal cord nociceptive responses and increased the expression of IL-6, which was used here as an inflammatory marker, in the NP cells. Moreover, the present results demonstrated that miR-223, miR-760 and miR145 may be up-regulated in NP and released in exosomelike vesicles (ELV) when the NP tissue is exposed to the dorsal nerve roots. In particular, the NP expression of miR-223, which had an anti-nociceptive effect at the spinal level, was increased.

In serum samples from our patients with lumbar radicular pain, the extracellular miR-223 expression was higher when the patients arrived at the clinic than it was 12 months later. Although no clear relationship between miR-223 and IL- 6 was observed, a correlation between the change in the miR-223 levels and change in the pain scores was demonstrated. In addition, high levels of miR-223 in the acute phase after disc herniation were associated with a decreased risk of chronic lumbar radicular pain.

These observations suggests that disc herniation also involved changes in miR-223 release. Moreover, herni0ated discs produce chemotactic factors that promote the recruitment of macrophages and T-cells to the disc [7]. Earlier observations show that miR-223 expressed in the myeloid lineage may be an important modulator of myeloid differentiation and inflammatory responses [33]. In activated macrophages, miR-223 reduces the production of IL- 6 and NO [34]. The down-regulation of miR223 , in contrast, has been reported to increase the release of IL-6 and IL- $1 \beta$ [35].

Recent clinical data demonstrate that persistent pain after disc herniation may be associated with low-grade systemic inflammation [10]. An important regulator of inflammatory cytokines is nuclear factor kappa-lightchain-enhancer of activated B cells (NF- $k \beta)$. Interestingly, miR-223 can inhibit NF- $k \beta$ activation and down-stream signalling including the activation of macrophages/ immune cells [34, 36]. In patients with rheumatoid arthritis, miR-223 appears to increase in response to anti-TNF treatment [28]. Moreover, miR-223 may be negatively correlated with inflammatory mediators [28], suggesting that miR-223 could be involved in a negative feedback loop that decreases the cytokines synthesis.

In this process, miR-223, similar to other miRs, can be exchanged between cells via exosomes (Additional file 3). These small vesicles, typically $40-100 \mathrm{~nm}$, may be secreted by several cell-types including NP cells. Earlier data have suggested that exosomes transfer miRs to recipient cells where they repress resident target mRNA translation [20]. Therefore, the up-regulation and release of miR-223 from the NP cells close to the nerve roots could influence on the activity in nearby neuronal tissues, i.e., 
a
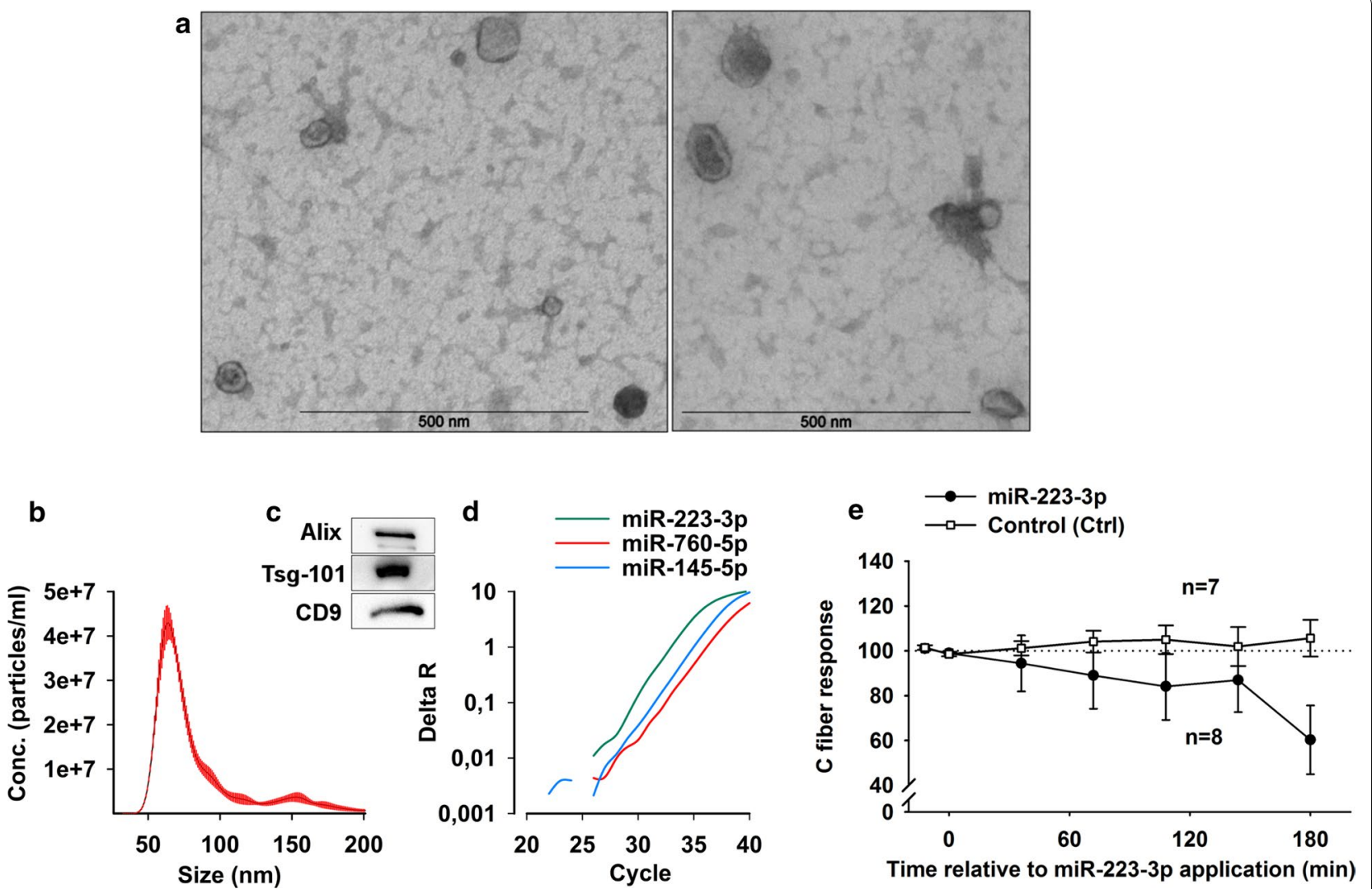

Fig. 4 a Identification of exosome-like vesicles (ELVs). The morphology of the ELVs was inspected by electron microscopy. NP was excised from the tail of rats and incubated in serum-free media prior to isolation of ELVs by ultracentrifugation. $\mathbf{b}$ The size distribution of the ELV population was measured by NTA. Error bars are shown in red. c Several exosome-associated proteins were detected in the ELV fraction by western blot. Representative western blot showing alix, tsg101 and CD-9. d Examples of miR-223, miR-760 and miR-145 qPCR amplification plot in the exosome fraction. e Electrically evoked C-fiber responses in the dorsal horn neurons at baseline i.e., before and 180 min after the application of miR-223 onto the dorsal nerve roots; within subjects effects rmANOVA, $p=0.037$. Data are given as the mean $\pm S E M$

the DRG or spinal cord. Moreover, previous data show that miR-223 through the NR2B subunits in neuronal tissue may inhibit NMDA induced $\mathrm{Ca}^{2+}$ influx [37].

Therefore, miR-223 transferred to recipient cells in the DRG or spinal cord could, as demonstrated in the present study, inhibit nociceptive signalling at the spinal level. Our observation that extracellular miR-223 expression was higher in patients who recovered than those who developed persistent pain, supports this hypothesis. Moreover, profiling studies have demonstrated down-regulation of miRs in the DRG in rats following inflammatory muscle pain [38]. However, there is a debate over how these animal findings translates to patients. Hence, more research is needed to determine the functional role of miR-223 in lumbar radicular pain patients.

\section{Conclusions}

Previous findings show that lumbar radicular pain after disc herniation is associated with up-regulation of inflammatory mediators $[9,10]$. The present animal data demonstrated that this process may involve release of 

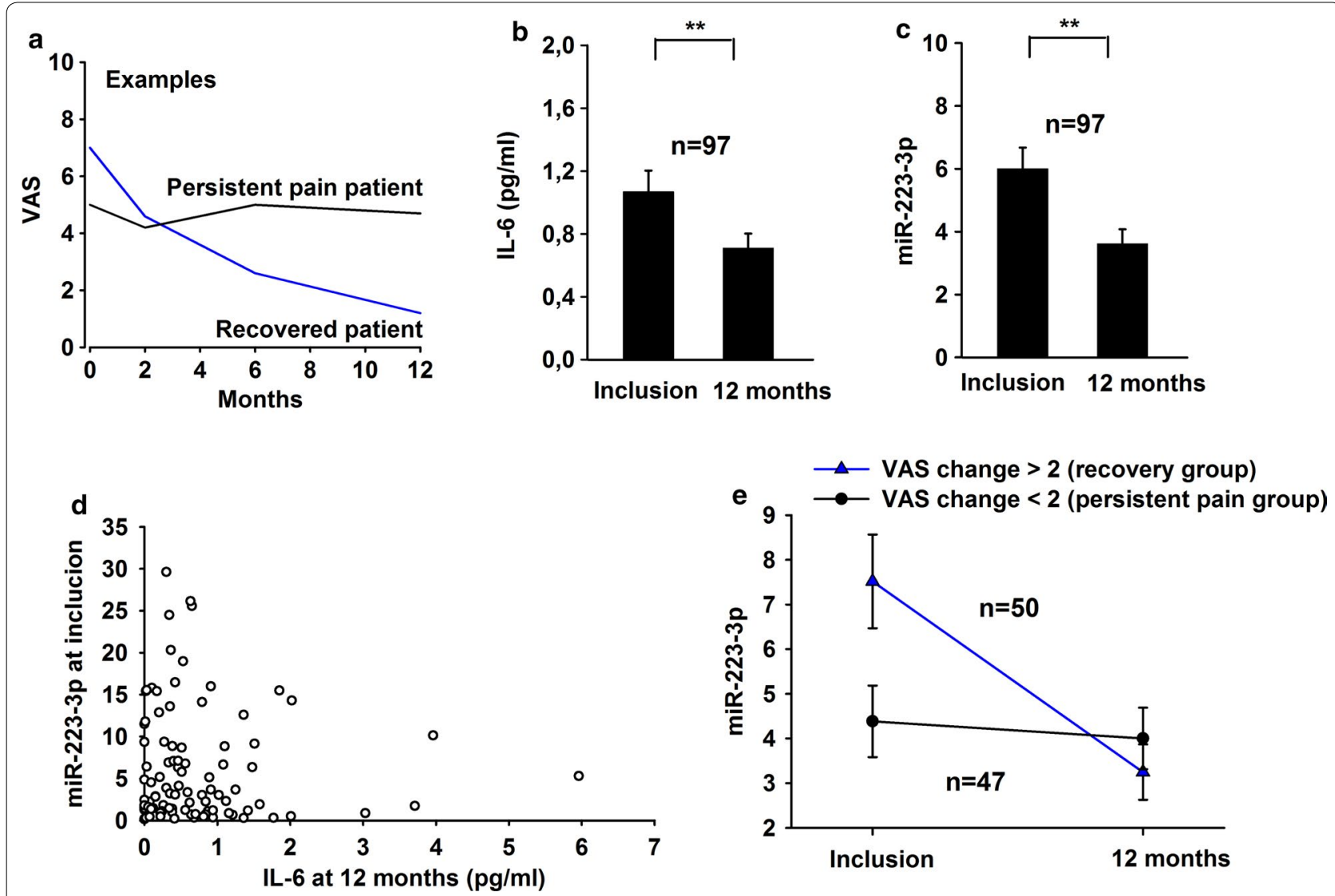

Fig. 5 a Typical examples of the VAS scores of two patients after disc herniation. $\mathbf{b}, \mathbf{c} \| \mathrm{L}-6$ and miR-223 levels in the serum of patients with lumbar radicular pain at inclusion and 12 months. Paired Students $t$ test, ${ }^{* *} \mathrm{p}<0.01$. d miR-223 at inclusion versus IL- 6 at 12 months in the serum of patients with lumbar radicular pain. e miR-223 levels at inclusion in the recovery group versus the persistent pain group defined as reduction in VAS from inclusion to 12 months. Linear regression adjusted for the sex, age and smoking, [beta $=-2.97, \mathrm{p}=0.031,95 \% \mathrm{Cl}(-5.67,-0.27)]$

Table 2 Characteristics of patients grouped in recovery and persistent pain group

\begin{tabular}{lccc}
\hline & Recovery group $\mathbf{n}=\mathbf{5 0}$ & Persistent pain group $\mathbf{n}=\mathbf{4 7}$ & $\boldsymbol{p}$ value \\
\hline Gender, men/women (\%) & $22 / 28(44 / 56)$ & $18 / 29(38 / 62)$ & $\mathrm{n} \cdot \mathrm{s}^{\mathrm{a}}$ \\
Mean age (min-max) & $40(22-58)$ & $42(21-59)$ & $\mathrm{n} \cdot \mathrm{s}^{\mathrm{b}}$ \\
Current smoker, yes/no (\%) & $15 / 35(30 / 70)$ & $19 / 28(40 / 60)$ & $\mathrm{n} \cdot \mathrm{s}^{\mathrm{a}}$ \\
Treatment, surgery/conservative (\%) & $23 / 27(46 / 54)$ & $13 / 34(28 / 72)$ & $<0.05^{\mathrm{a}}$ \\
\hline
\end{tabular}

The recovery group was defined by a reduction in VAS (VAS inclusion-VAS 12 months) $>2.0$, whereas the persistent pain group was defined by a reduction in VAS (VAS inclusion-VAS 12 months) $<2.0$

n.s not significant

a Pearson Chi square

b Unpaired Student's t test

small non-coding RNAs including miR-223 in ELVs. An anti-nociceptive effect of miR-223 at the spinal level was also demonstrated. However, increased levels of the miR223 after disc herniation was only observed in patients who recovered. Hence, dysregulation of miR-223 in the acute phase after disc herniation may be associated with persistent lumbar radicular pain.

\section{Additional files}

Additional file 1: Table S1. Additional table.

Additional file 2: Figure S1. Additional figure.

Additional file 3. Additional datas for Figs 1, 2, 3, 4 and 5. 


\section{Abbreviations}

DRG: dorsal root ganglion; ELV: exosome-like vesicles; miR: microRNA; NP: nucleus pulposus; IL-6: interleukin-6; NO: nitric oxide; PGE2: prostaglandin E2; MMP: matrix metalloproteinase; IL-1 $\beta$ : interleukin-1 beta; TNF: tumour necrosis factor; VAS: visual analogue scale; NF-kß: nuclear factor kappa-light-chainenhancer of activated B cells; ELISA: enzyme-linked immunosorbent assay; rmANOVA: repeated measures analysis of variance.

\section{Authors' contributions}

AM, DJ, SP, FH, CR and JG designed the research; AM, DJ, SP, FH and JG performed the research; AM, DJ, SP, CR and JG analysed the data; and AM and JG wrote the paper. All authors read and approved the final manuscript.

\section{Author details}

${ }^{1}$ National Institute of Occupational Health, Pb 8149 Dep., 0033 Oslo, Norway.

${ }^{2}$ Department of Physical Medicine and Rehabilitation, Oslo University

Hospital, Oslo, Norway. ${ }^{3}$ Faculty of Medicine, University of Oslo, Oslo, Norway.

${ }^{4}$ Department of Biosciences, University of Oslo, Oslo, Norway.

\section{Acknowledgements}

We thank Elina I. Schistad for collecting patient samples and clinical measurements at Ullevål University Hospital and Andreas Brech at The Norwegian Radium Hospital for assistance with electron microscopy. We also thank Tiril Schjølberg, Aqsa Mahmood and Øivind Skare at National Institute of Occupational Health for technical support.

\section{Competing interests}

The authors declare that they have no competing interests.

\section{Availability of data and materials}

All data will be available on a additonal file.

\section{Ethics}

All animal experiments were approved by the Norwegian Animal Research Authority (NARA 2015/257472). The clinical part of the study was approved by the Norwegian Social Science Data Service and by Norwegian Regional Committee for Medical Research Ethics (REK 2014/1725).

\section{Funding}

This study received financial support from the South Eastern Norway Regional Health Authority, Grant Number 2013/20589.

\section{Publisher's Note}

Springer Nature remains neutral with regard to jurisdictional claims in published maps and institutional affiliations.

Received: 25 November 2016 Accepted: 19 April 2017

Published online: 01 May 2017

\section{References}

1. Olmarker K, Rydevik B, Nordborg C. Autologous nucleus pulposus induces neurophysiologic and histologic changes in porcine cauda equina nerve roots. Spine (Phila Pa 1976). 1993;18(11):1425-32.

2. Kayama S. Incision of the anulus fibrosus induces nerve root morphologic, vascular, and functional changes: an experimental study. Spine (Phila Pa 1976). 1996;21(22):2539.

3. Anzai H, Hamba M, Onda A, Konno S, Kikuchi S. Epidural application of nucleus pulposus enhances nociresponses of rat dorsal horn neurons. Spine. 2002;27(3):E50-5.

4. Cuellar JM, Montesano PX, Antognini JF, Carstens E. Application of nucleus pulposus to $L 5$ dorsal root ganglion in rats enhances nociceptive dorsal horn neuronal windup. J Neurophysiol. 2005;94(1):35-48.

5. Takebayashi T, Cavanaugh JM, Cuneyt Ozaktay A, Kallakuri S, Chen C. Effect of nucleus pulposus on the neural activity of dorsal root ganglion. Spine (Phila Pa 1976). 2001;26(8):940-5.
6. Kang JD, Stefanovic-Racic M, McIntyre LA, Georgescu HI, Evans CH. Toward a biochemical understanding of human intervertebral disc degeneration and herniation: contributions of nitric oxide, interleukins, prostaglandin E2, and matrix metalloproteinases. Spine. 1997:22(10):1065-73.

7. Doita M, Kanatani T, Ozaki T, Matsui N, Kurosaka M, Yoshiya S. Influence of macrophage infiltration of herniated disc tissue on the production of matrix metalloproteinases leading to disc resorption. Spine (Phila Pa 1976). 2001;26(14):1522-7.

8. Takada T, Nishida K, Doita M, Miyamoto H, Kurosaka M. Interleukin-6 production is upregulated by interaction between disc tissue and macrophages. Spine (Phila Pa 1976). 2004;29(10):1089-92 (discussion 93).

9. Pedersen LM, Schistad E, Jacobsen LM, Røe C, Gjerstad J. Serum levels of the pro-inflammatory interleukins $6(\mathrm{IL}-6)$ and $-8(\mathrm{IL}-8)$ in patients with lumbar radicular pain due to disc herniation: a 12-month prospective study. Brain Behav Immun. 2015;46:132-6. doi:10.1016/j.bbi.2015.01.008.

10. Moen A, Lind A-L, Thulin M, Kamali-Moghaddam M, Røe C, Gjerstad $J$, et al. Inflammatory serum protein profiling of patients with lumbar radicular pain one year after disc herniation. Int J Inflamm. 2016;2016:8. doi:10.1155/2016/3874964.

11. Jonas $\mathrm{S}$, Izaurralde E. Towards a molecular understanding of microRNAmediated gene silencing. Nat Rev Genet. 2015;16(7):421-33. doi:10.1038/ nrg3965.

12. Wang CY, Yang SH, Tzeng SF. MicroRNA-145 as one negative regulator of astrogliosis. Glia. 2015;63(2):194-205. doi:10.1002/glia.22743.

13. Gu SX, Li X, Hamilton JL, Chee A, KC R, Chen D, et al. MicroRNA-146a reduces IL-1 dependent inflammatory responses in the intervertebral disc. Gene. 2015;555(2):80-7. doi:10.1016/j.gene.2014.10.024.

14. Liu G, Cao P, Chen H, Yuan W, Wang J, Tang X. MiR-27a regulates apoptosis in nucleus pulposus cells by targeting PI3K. PLOS ONE. 2013;8(9):e75251. doi:10.1371/journal.pone.0075251.

15. Wang HQ, Yu XD, Liu ZH, Cheng X, Samartzis D, Jia LT, et al. Deregulated miR-155 promotes Fas-mediated apoptosis in human intervertebral disc degeneration by targeting FADD and caspase-3. J Pathol. 2011;225(2):232-42. doi:10.1002/path.2931.

16. Liu H, Huang X, Liu X, Xiao S, Zhang Y, Xiang T, et al. miR-21 promotes human nucleus pulposus cell proliferation through PTEN/AKT signaling. Int J Mol Sci. 2014;15(3):4007-18. doi:10.3390/ijms15034007.

17. Yu X, Li Z, Shen J, Wu WK, Liang J, Weng X, et al. MicroRNA-10b promotes nucleus pulposus cell proliferation through RhoC-Akt pathway by targeting HOXD10 in intervetebral disc degeneration. PLOS ONE. 2013:8(12):e83080. doi:10.1371/journal.pone.0083080.

18. Ismail N, Wang Y, Dakhlallah D, Moldovan L, Agarwal K, Batte K, et al. Macrophage microvesicles induce macrophage differentiation and miR-223 transfer. Blood. 2013;121(6):984-95. doi:10.1182/blood-2011-08-374793.

19. Nolte EN, Buermans HP, Waasdorp M, Stoorvogel W, Wauben MH, AC't Hoen P. Deep sequencing of RNA from immune cell-derived vesicles uncovers the selective incorporation of small non-coding RNA biotypes with potential regulatory functions. Nucleic Acids Res. 2012;40(18):927285. doi:10.1093/nar/gks658

20. Montecalvo A, Larregina AT, Shufesky WJ, Stolz DB, Sullivan ML, Karlsson $J M$, et al. Mechanism of transfer of functional microRNAs between mouse dendritic cells via exosomes. Blood. 2012;119(3):756-66. doi:10.1182/ blood-2011-02-338004.

21. Valadi H, Ekstrom K, Bossios A, Sjostrand M, Lee JJ, Lotvall JO. Exosomemediated transfer of mRNAs and microRNAs is a novel mechanism of genetic exchange between cells. Nat Cell Biol. 2007;9(6):654-9. doi:10.1038/ncb1596.

22. Fabbri M, Paone A, Calore F, Galli R, Gaudio E, Santhanam R, et al. MicroRNAs bind to toll-like receptors to induce prometastatic inflammatory response. Proc Natl Acad Sci USA. 2012;109(31):E2110-6. doi:10.1073/ pnas.1209414109.

23. Park CK, Xu ZZ, Berta T, Han Q, Chen G, Liu XJ, et al. Extracellular microRNAs activate nociceptor neurons to elicit pain via TLR7 and TRPA1. Neuron. 2014;82(1):47-54. doi:10.1016/j.neuron.2014.02.011.

24. Friedman RC, Farh KK, Burge CB, Bartel DP. Most mammalian mRNAs are conserved targets of microRNAs. Genome Res. 2009;19(1):92-105. doi:10.1101/gr.082701.108. 
25. Andersen HH, Duroux M, Gazerani P. Serum MicroRNA signatures in migraineurs during attacks and in pain-free periods. Mol Neurobiol. 2016;53(3):1494-500. doi:10.1007/s12035-015-9106-5.

26. Orlova IA, Alexander GM, Qureshi RA, Sacan A, Graziano A, Barrett JE, et al. MicroRNA modulation in complex regional pain syndrome. J Transl Med. 2011;9(1):1-11. doi:10.1186/1479-5876-9-195.

27. Bjersing JL, Bokarewa MI, Mannerkorpi K. Profile of circulating microRNAs in fibromyalgia and their relation to symptom severity: an exploratory study. Rheumatol Int. 2015;35(4):635-42. doi:10.1007/s00296-014-3139-3.

28. Castro-Villegas C, Perez-Sanchez C, Escudero A, Filipescu I, Verdu M, Ruiz-Limon $P$, et al. Circulating miRNAs as potential biomarkers of therapy effectiveness in rheumatoid arthritis patients treated with anti-TNFalpha. Arthritis Res Ther. 2015;17:49. doi:10.1186/s13075-015-0555-z.

29. Pedersen LM, Jacobsen LM, Mollerup S, Gjerstad J. Spinal cord longterm potentiation (LTP) is associated with increased dorsal horn gene expression of IL-1 beta, GDNF and iNOS. Eur J Pain. 2010;14(3):255-60. doi:10.1016/j.ejpain.2009.05.016.

30. Egeland N, Moen A, Pedersen LM, Røe C, Gjerstad J. Spinal nociceptive hyperexcitability induced by experimental disc herniation is associated with enhanced local expression of Csf1 and FasL. Pain. 2013;154:1743-8.

31. Jacobsen DP, Moen A, Haugen F, Gjerstad J. Hyperexcitability in spinal WDR neurons following experimental disc herniation is associated with upregulation of fractalkine and its receptor in nucleus pulposus and the dorsal root ganglion. Int J Inflam. 2016;2016:6519408. doi:10.1155/2016/6519408.

32. Phuyal S, Skotland T, Hessvik NP, Simolin H, Overbye A, Brech A, et al. The ether lipid precursor hexadecylglycerol stimulates the release and changes the composition of exosomes derived from PC-3 cells. J Biol Chem. 2015;290(7):4225-37. doi:10.1074/jbc.M114.593962.
33. Johnnidis JB, Harris MH, Wheeler RT, Stehling-Sun S, Lam MH, Kirak O, et al. Regulation of progenitor cell proliferation and granulocyte function by microRNA-223. Nature. 2008;451(7182):1125-9. doi:10.1038/ nature06607.

34. Wang J, Bai X, Song Q, Fan F, Hu Z, Cheng G, et al. miR-223 inhibits lipid deposition and inflammation by suppressing toll-like receptor 4 signaling in macrophages. Int J Mol Sci. 2015;16(10):24965-82. doi:10.3390/ ijms161024965.

35. Chen Q, Wang H, Liu Y, Song Y, Lai L, Han Q, et al. Inducible microRNA-223 down-regulation promotes TLR-triggered IL-6 and IL-1 beta production in macrophages by targeting STAT3. PLoS ONE. 2012;7(8):e42971. doi:10.1371/journal.pone.0042971.

36. Liu Y, Wang R, Jiang J, Yang B, Cao Z, Cheng X. miR-223 is upregulated in monocytes from patients with tuberculosis and regulates function of monocyte-derived macrophages. Mol Immunol. 2015;67(2 Pt B):475-81. doi:10.1016/j.molimm.2015.08.006.

37. Harraz MM, Eacker SM, Wang X, Dawson TM, Dawson VL. MicroRNA-223 is neuroprotective by targeting glutamate receptors. Proc Natl Acad Sci USA. 2012;109(46):18962-7. doi:10.1073/pnas.1121288109.

38. Bai G, Ambalavanar R, Wei D, Dessem D. Downregulation of selective microRNAs in trigeminal ganglion neurons following inflammatory muscle pain. Mol Pain. 2007;3:15. doi:10.1186/1744-8069-3-15.

\section{Submit your next manuscript to BioMed Central and we will help you at every step:}

- We accept pre-submission inquiries

- Our selector tool helps you to find the most relevant journal

- We provide round the clock customer support

- Convenient online submission

- Thorough peer review

- Inclusion in PubMed and all major indexing services

- Maximum visibility for your research

Submit your manuscript at www.biomedcentral.com/submit
() Biomed Central 